\title{
A first for ovarian cancer
}

Ovarian cancer can be effectively treated if caught early, but it is generally diagnosed after it has metastasized. This has a serious impact on the survival of patients, and has also limited our knowledge of the early genetic changes that induce ovarian cancer. Sandra Orsulic et al., reporting in the launch issue of Cancer Cell, have developed the first mouse model of ovarian cancer with defined genetic lesions, which should improve our understanding of cancer development.

$\operatorname{Tr} p 53^{+/+}$and $\operatorname{Tr} p 53^{-/-}$ovarian cells from transgenic mice that express an avian virus receptor were transfected in vitro with avian retroviral vectors carrying oncogenes that are frequently amplified or mutated in human ovarian cancer: Kras2, c-Myc and Akt. The authors investigated which combinations of genetic lesions could induce tumour formation in vivo by injecting these ovarian cells subcutaneously into nude mice and monitoring for tumour growth. Tumours arose only from cells that were deficient for $\operatorname{Tr} p 53$, and had acquired at least two oncogenes by infection.
But can these cells also induce tumour growth at their natural site of formation: the ovaries? Implantation of infected cells under the ovarian capsule of nude mice results in ovarian tumours within 2 weeks. These metastasize to the same sites as human ovarian cancers within 4 weeks.

As the immune system might limit tumour growth in humans, the infected ovarian cells were also implanted under the ovarian capsule of the immunocompetent mice from which the cells were removed; ovarian tumours developed after three months.

This mouse model should help unravel the molecular basis of ovarian cancer development, and will provide a system for testing new therapeutic approaches.

\section{Emma Greenwood}

(2) References and links

ORIGINAL RESEARCH PAPER Orsulic, S. et al. Induction of ovarian cancer by defined multiple genetic changes in a mouse model system. Cancer Cell 1, 1-10 (2002) model system. WEB SITES
Harold Varmus's lab:

http://www.ski.edu/lab homepage.cfm?lab=203 Encyclopedia of Life Sciences: http://www.els.net human disease: mouse models

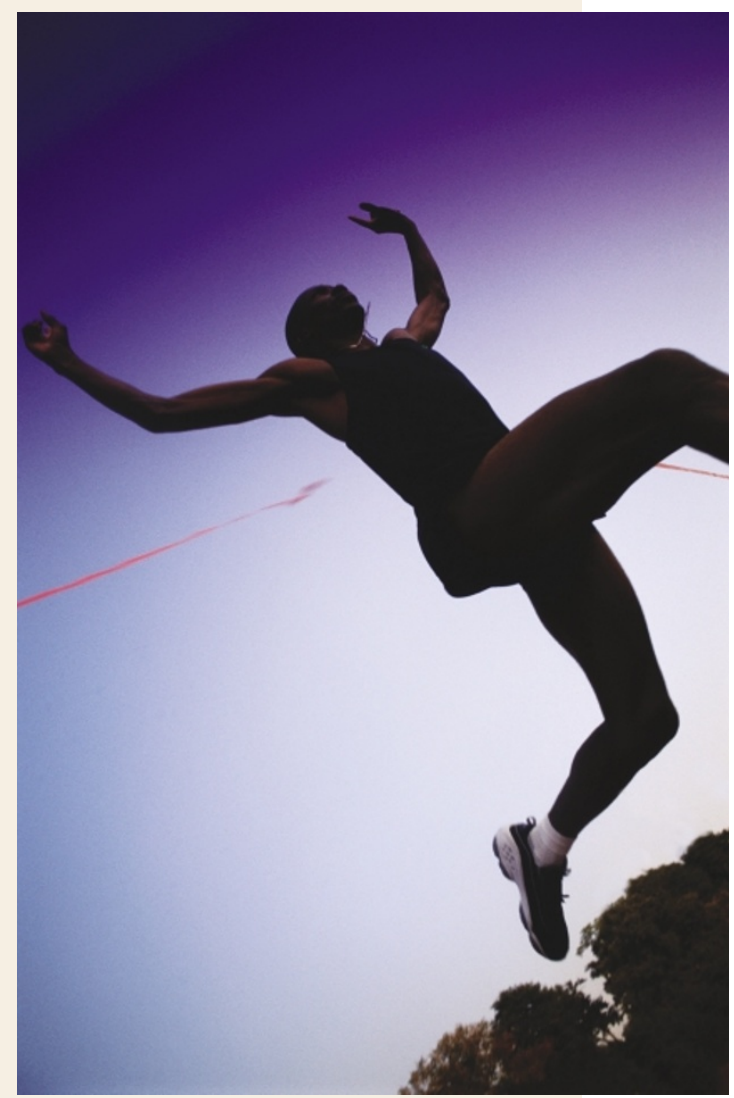

TUMOUR SUPPRESSORS

\section{Keeping damage in check}

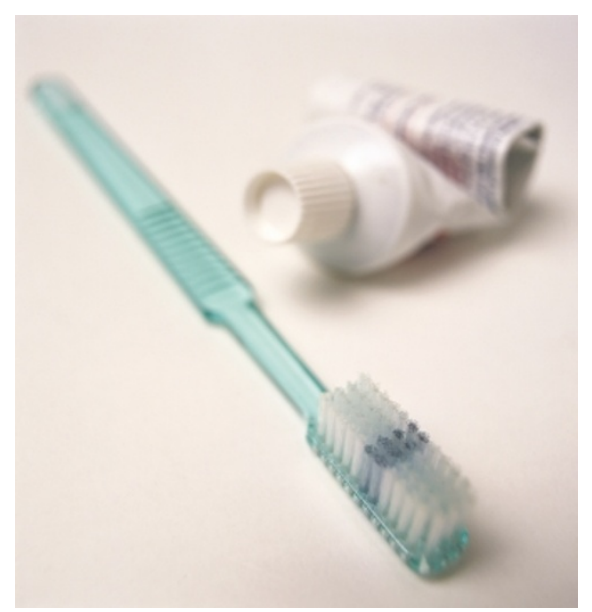

Mutations in the tumour-suppressor gene $B R C A 1$ predispose individuals to breast and ovarian cancer. The protein has been assigned many functions though, so how does its deficiency contribute to tumorigenesis? Ronit Yarden et al. report in Nature Genetics that BRCA1 is involved in cell-cycle arrest following DNA damage, which allows cells time to repair their DNA.
The breast cancer cell line HCC1937 possesses just one copy of $B R C A 1$, and this contains an inactivating mutation that is associated with an increased cancer risk. These cells are unable to arrest following $\gamma$-irradiation, but transfection with $B R C A 1$ restores this ability.

As cells arrest at the $\mathrm{G} 2 / \mathrm{M}$ checkpoint by inhibiting the $\mathrm{CDC} 2-\mathrm{cyclin}-\mathrm{B}$ kinase, Yarden et al. determined the protein levels and activity of CDC2-cyclin-B in HCC1937 cells and those transfected with BRCA1. Both expression of cyclin $\mathrm{B} 1$ and activity of the CDC2-cyclin-B kinase are decreased in BRCA1-expressing cells.

CDC2-cyclin-B activity is negatively regulated by the phosphorylation of CDC2's Tyr15 residue, so are the proteins that control Tyr 15 phosphorylation also regulated by BRCA1? $\mathrm{CDC} 25 \mathrm{C}$, the activatory phosphatase, is downregulated after irradiation in BRCA1-expressing cells, and this corresponds with an increase in the inhibitory kinase WEE1 and the level of phosphorylated CDC2-Tyr 15. 14-3-3 proteins, which transport $\mathrm{CDC} 25 \mathrm{C}$ from the nucleus following DNA damage to prevent activation of CDC2-cyclin-B, are also upregulated in BRCA1expressing cells, and immunofluorescence revealed that $\mathrm{CDC} 25 \mathrm{C}$ is, indeed, cytoplasmic in BRCA1-expressing cells.

But how does BRCA1 induce these effects? $\mathrm{CHK} 1$ and $\mathrm{CHK} 2$ are essential for the cell-cycle arrest in response to DNA damage, so might BRCA1 activate one of these checkpoint proteins? BRCA1 physically interacts with CHK1 and stimulates its activity after DNA damage. The G2/M arrest is also dependent on CHK1, as BRCA1expressing cells that were treated with a CHK1 inhibitor lost the ability to induce this arrest.

BRCA1 therefore seems to be the link between the DNA-damage-sensing proteins ATM and ATR - which phosphorylate BRCA 1 - and downstream members of the G2/M checkpoint pathway. So BRCA1 maintains genome stability by allowing cells time to repair their DNA following DNA damage. Only time will tell if it also inhibits tumorigenesis by other mechanisms.

Emma Greenwood

\section{(2) References and links}

ORIGINAL RESEARCH PAPER Yarden, R. I. et al. BRCA regulates the G2/M checkpoint by activating Chk1 kinase upon DNA damage. Nature Genet. 30, 285-289 (2002) WEB SITE

Lawrence Brody's lab:

http://www.nhgri.nih.gov/Intramural_research/People/brody.htm 